\title{
Research on the Architecture and Storage Optimization Idea and the Applications on the Computer Software Development
}

\author{
Jinyu Wang \\ Hohhot Vocational College, Hohhot 010051, China.
}

\begin{abstract}
Keywords: Computer Software, Storage Optimization, Idea, Architecture and Storage.
\end{abstract}
\begin{abstract}
This paper conducts research on the architecture and the storage optimization idea and the applications on the computer software development. High energy consumption is not only the increase of the storage system itself consumption cost, also adds to the cost of general additional consumption. For example, high quantity of heat produced by high energy consumption not only increases the energy consumption of the cooling system, also make the storage density can't improve storage devices, reduces the space utilization, these all add to the cost of the data center. The development of the reconfigurable computing technology that make the computer not only has the traditional architecture based on instruction stream computation and based on data stream computing architecture, and based on the structure of flow architecture. This paper analyzes the issues from a novel perspective that will enhance the performance of the methodology.
\end{abstract}

\section{Introduction}

Architecture software simulation technology way to use the software to simulate the function of the computer system hardware structure level in the system and performance characteristics and in the design and manufacture of general modern computer systems, software simulation technology using architecture to the architecture plan evaluation and validation has become an indispensable part. The development of the reconfigurable computing technology that make the computer not only has the traditional architecture based on instruction stream computation and based on data stream computing architecture, and based on the structure of flow architecture. So, in order to more clearly to describe classifying system structure of the modern computer, we need to have a new computer architecture classification model. The listed ones are the essential issues.

- Based on the structure makes the flow calculation of architecture. Along with the further increase of chip integration, the realization of the hardware from reconstruction, development represented by PLD and FPGA to various granular form of static reconfigurable hardware implementation, further improve the reusability of the hardware.

- Based on the instruction stream computing architecture. Based on the instruction stream computing architecture is mainly of the von Neumann computer architecture. The operation instruction is consists of two parts, operation code and address code data, so the instruction stream includes operating flow and data flow that is working on the control flow principle which is one of the most flexible architecture.

- Based on data stream computing architecture. The simplest architecture is based on the data stream computation ASIC circuit, because ASIC circuit to complete the function is fixed, don't need operation code to define the function to be completed, only need to use the address code to indicate the source of the ASIC circuit of input data and output the results of the destination that can complete the ASIC itself, as defined by the calculation function.

At present, no unified definition of efficient storage, the storage vendor respectively have their own definitions and interpretation, but the target is the basic consistent, namely to improve storage efficiency, simplify the storage management, reduce energy consumption of storage, to obtain lower total holding costs and operating costs. Because of some although the capacity of the storage system and application system have high reliability requirements, but the performance is not strict, a lot of disk parallel storage system can improve data transfer rate, however, in the case of low I/O load, this 
kind of high performance cannot be make full use of, so can reduce the number of the parallel disk, decrease the storage performance of the system to achieve energy saving. The experiment shows that the section storage systems have achieved good effect. To deal with the mentioned challenges, in the later sections, we will discuss the issues in detail.

\section{The Proposed Methodology}

\subsection{Computer Architecture Principles.}

It is the basis of reconfigurable reusable resources. In the FPGA, reconfigurable computing system with the method of restructuring, the reuse of resources is the functional unit; FPGA appears, make possible a refactoring, the reuse of resources is the basic door and line, through a configuration file, define the nature of each door and line connection that change the function of the hardware. Computer network computer system is the basic characteristic of members can work independently, so the control logic is scattered in the whole system, each member logically share a single computer system, and the width of the memory address space and the whole hardware system is managed by a single operating system while in terms of architecture, the computer system for at least the following a few questions. (1) Due to the size of the process is differ, the relative speed of execution process is not clear. Process synchronization between the general communication problems between the became process problems. Synchronous mechanism is usually based on the principle of Shared variables or messaging. Multiprocessor systems respectively called tight FGC accordingly with loose FGC system. (2) In the ideas of the implicit call, as parts are no longer a processes called directly, but a statement of one or more events. The other components in the system can through a linked to this or these events of this process, in this or these events to register. When the event is declared, the system will automatically call all in this process or registered in these events. (3) Future of the software engineering design will gradually become like mechanical and architectural design of mechanical assembly process, so as to get rid of the hard mental work and beyond by quality assurance manual labor. The software architecture will play a leading role. (4) Like assembly line with vector machine (SVM), in the Shared memory multiprocessor system also exists memory access conflict, resulting in the access latency which can be shown as the figure 1 .

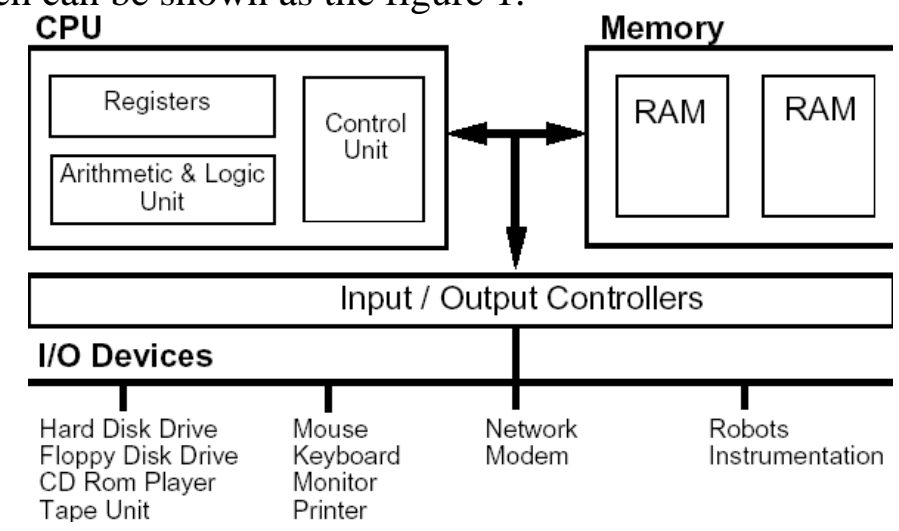

Fig. 1 The Classic Computer Architecture

Because the calculation function of PU array is in advance in accordance with the algorithm need to design good, do not need to control the PU array to the command, and only need to command control data memory data out of the PU array, which is on the PU array as is the work flow calculation method according to the data, do not need to have the operation flow the function, and only need to have data flow function. Therefore, the PU control array of work orders, not like the usual commands that control data flow and operation flow, and only the control data flow function. In essence, it is now known as the origin of flow.

\subsection{Software Development Methodologies and Approaches.}

After decades of software engineering practice, a variety of the software development method has been widely used. A software development method is using the technology and the symbol defined to organize the method of software production process. Software development method is one of the 
important contents of general software engineering theory, in software development methods, for the development of software "to do" and "how to do" that gives a clear and detailed answer.

Early software development activities of the individual characteristics of very severe, write a program to make the development of the lack of unified standards, application programmers write follow one's inclinations that cause the readability of the program, clarity, understandability is poorer, software maintenance work to bring huge difficulties. The root of this phenomenon has the certain relationship with the scale of software development: due to the time of the software on a smaller scale, the development process is simpler, people make the mistake of thinking that develop software just to write programs, ignore the other software development program. For dealing with this general and basic challenge, the corresponding solutions are proposed for further optimization.

- Specification describes DSSA. This stage by using appropriate formal specification defines the DSSA, mathematics model and the formal specification DSSA is described, in order to make the precise description of it, without ambiguity.

- Refinement and verification of general DSSA. DSSA is through from abstract to concrete, incremental refinement. In the process of refinement of DSSA, need for different levels of abstraction DSSA verification, to determine whether a specific DSSA agree with more abstract DSSA semantics, and can realize the abstract DSSA.

- According to the field demand determine the DSSA, which meet the demand of field is given by the artifacts and the connection between components, constraints the system architecture.

- Systems analysts can quickly establish the demand model of the current system. That is to say, the domain model will help the developers to understand an application domain, and can be used as the starting point of system analysis. Once the demand model of the current system, to use the traceability between the requirements model and DSSA.

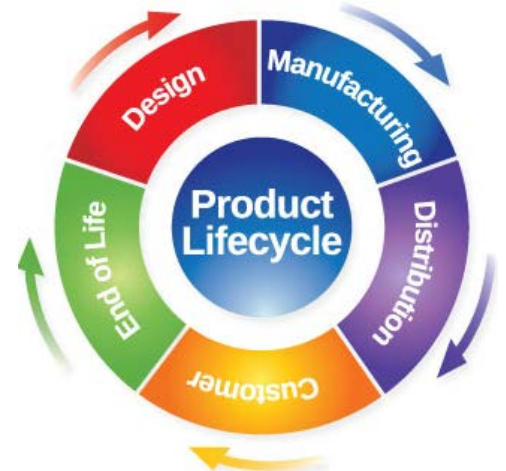

Fig. 2 The Software Development Methodologies and Steps

\subsection{Computer Storage Optimization.}

With the continuous development of data acquisition technology and a large number of the data processing of the new technology into use, the flow of data to deal with geometric base growth, the original IBM cluster computer storage subsystem has been unable to meet the needs of processing production, scientific research on storage capacity, and vast amounts of IO data exchange bottleneck will behave more and more outstanding, affects the speed of processing production. We can improve the performance of the unit disk in the disk in the running state of the same number of cases, improve the overall performance storage system as similarly, in the application system of the storage system performance requirements, under the condition of same can close more disk and make whole storage system energy saving effect is more significant.

The following three problems in the current implementation. (1) Data transmission between the hardware and software process still needs general processor control, the burden of aggravating the GPP, and the data transmission is limited by the speed of the GPP access to data. (2) Support for hardware process system services is provided by means of the hardware module, not easy to use and difficult to expand. (3) Does not support Shared storage platform to support process communication mode based on Shared storage. As a result of the design for reconfigurable platform for distributed storage, so the process of hardware and the other part of the communication system based on message 
passing way, the efficiency is low. Storage medium is different, according to the ontology of the ontology based on memory storage method is roughly divided into storage, plain text storage method, based on the database storage and 4 kinds of specialized management tool way, the most studied, the most widely used is based on database storage.

\section{Stability of the Software}

Pressure testing of software refers to the module of the test is exert enormous work load, check the software module in the work condition of heavy load or even load limit. On the one hand, can the stability of the test module is accord with the requirement of actual operation environment, on the other hand through the extreme pressure test can also calculate the software stability limit value, through the value regulation in the process of actual operation safe use of the software. Complex network theory with a strong interdisciplinary characteristics and its study involves all kinds of fields. Studies have shown a complex network phenomenon also exists in large software system while the topological structure of complex software systems can also use the form of network as the function of the software system, performance and stability to a large extent influenced by software topology structure while so, from the perspective of complex network to software engineering, using complex network to explore the structure of the large-scale software systems for the tool.

- It is a function of amplitude threshold value, which indicates that in the test when the function of software affected more than the set value, judgment software to malfunction. The higher the threshold value is set, the longer the software stability may, setting is lower, the shorter the plateau may.

- High stability refers to the maturity system under high load as low stability refers to maturity under low load. High stability and low stability can be applied in collapse risk analysis, in view of the different results, take different measures to improve the stability of the software, reduce the risk of software failure.

- The higher anti-interference ability stronger stability, anti-interference ability is weak, the lower the stability. This requests us in the process of test that needs to simulate some possible interference factors.

\section{Summary and Conclusion}

This paper conducts research on the architecture and the storage optimization idea and the applications on the computer software development. For the RAMCloud, memory capacity is one of the main constraints. The adoption of log structure RAMCloud segment of memory to store data, so when storing a large number of small files, each small file takes up the entire section, so that the segment fragments, effective utilization of memory fell sharply while although RAMCloud storage mechanism make its reliability, read and the write performance and bandwidth utilization have good balance, makes order file does not necessarily stored in the location of the continuous, even in the different master server. Under this basis, this paper integrates the general methodology of the storage optimization to solve the issues effectively.

\section{References}

[1]. Glass, A. M., and M. J. Cardillo. Holographic data storage. Eds. Hans J. Coufal, Demetri Psaltis, and Glenn T. Sincerbox. Vol. 76. Springer, 2012.

[2]. Jones, Chris B. Geographical information systems and computer cartography. Routledge, 2014.

[3]. Nelson, Bill, Amelia Phillips, and Christopher Steuart. Guide to computer forensics and investigations. Cengage Learning, 2014. 